\title{
Nanotechnology in pulp and paper industries: a review
}

\begin{abstract}
Cellulose and lignocellulose have great potential as nanomaterials because they are abundant, renewable, have a nanofibrillar structure, can be made multifunctional and self-assemble into well-defined architectures. New methods for liberating these materials, including nanodimensional cellulose fibrils, macromolecules and nanominerals will be needed in order to use the techniques developed for other nanomaterials as platforms for creating new woodbased materials and products. Research findings showed promising results in application of nanotechnology at different aspects of papermaking. Electrospinning; chemical treatment followed by mechanical techniques; or mechanical isolation methods were applied by different research groups to prepare cellulose nanofibres.
\end{abstract}

Keyword: Nanofibres; Nanotechnology; Paper; Pulp; Wood industry 